\title{
Aux sources des collections patrimoniales de la Monnaie de Paris
}

At the sources of the heritage collections of the Monnaie de Paris

\section{Béatrice Coullaré}

\section{(2) OpenEdition}

\section{Journals}

Édition électronique

URL : http://journals.openedition.org/artefact/2382

DOI : $10.4000 /$ artefact.2382

ISSN : 2606-9245

Éditeur :

Association Artefact. Techniques histoire et sciences humaines, Presses universitaires du Midi

Édition imprimée

Date de publication : 6 décembre 2018

Pagination : 321-327

ISBN : 978-2-8107-0595-5

ISSN : 2273-0753

Référence électronique

Béatrice Coullaré, «Aux sources des collections patrimoniales de la Monnaie de Paris », Artefact [En ligne], 8 | 2018, mis en ligne le 21 juin 2019, consulté le 10 décembre 2020. URL : http:// journals.openedition.org/artefact/2382 ; DOI : https://doi.org/10.4000/artefact.2382

\section{(c) $(1) \odot$}

Artefact, Techniques, histoire et sciences humaines est mise à disposition selon les termes de la Licence Creative Commons Attribution - Pas d'Utilisation Commerciale - Pas de Modification 4.0 International. 


\section{Aux sources des collections patrimoniales de la Monnaie de Paris}

\section{Béatrice Coullaré}

\section{Résumé}

La Monnaie des Médailles, qui fabriquait les médailles et les jetons des rois de France, conservait tous les outillages nécessaires à la frappe de ces objets dans son atelier. Quand Louis XIV nomma Nicolas Delaunay (1646-1727) « directeur du balancier des Médailles » en 1696, il lui demanda d'établir un inventaire détaillé. Ce travail de compilation dénotait, déjà à lépoque, l'intérêt suscité pour la valeur artistique et technique de ce fonds. Quand la Monnaie des Médailles fut réunie à la Commission des Monnaies en 1832, on décida de créer un musée monétaire. Installé dans les salons d'honneur de l'Hôtel des Monnaies, il reçut en dotation tous les outillages de médailles et de jetons hérités des souverains d'Ancien Régime. Sa mission était de servir de vitrine diplomatique pour promouvoir les savoir-faire artistiques et techniques des ateliers de fabrication. Aujourd'hui, le nouveau musée du 11 Conti expose soixante-dix poinçons et matrices en une présentation unique et didactique.

\section{Mots-clés}

médaille, technique, gravure, collection, musée, Monnaie des Médailles, Monnaie de Paris, savoir-faire, Nicolas Delaunay, Comte de Sussy, Dominique Vivant Denon

95 Béatrice Coullaré, « Aux sources des collections patrimoniales de la Monnaie de Paris », Artefact, 8, 2018, p. 321-327. 


\section{At the sources of the heritage collections of the Monnaie de Paris}

\section{Abstract}

The Mint of Medals, which made the medals and tokens of the kings of France, kept all the tools necessary to strike these objects in its workshop. When Louis XIV appointed Nicolas Delaunay (1646-1727) "directeur du balancier des Médailles" in 1696 , he asked him to establish a detailed inventory. This work of compilation denoted, already at the time, the interest aroused for the artistic and technical value of this fund. When the Mint of Medals was assembled at the Commission des Monnaies in 1832, it was decided to create a monetary museum. Housed in the salons of honor of the Hotel des Monnaies, he received in endowment all the tools of medals and tokens inherited from the rulers of Ancient Régime. Its mission was to serve as a diplomatic showcase to promote the artistic and technical know-how of manufacturing workshops. Today, the new museum of 11 Conti exhibits seventy punches and dies in a unique and didactic presentation.

\section{Keywords}

medal, technique, engraving, collection, museum, Monnaie des Médailles, Monnaie de Paris, know-how, Nicolas Delaunay, Comte de Sussy, Dominique Vivant Denon 


\section{Les archives métalliques des rois de France de Charles VIII à Louis XVI}

La Monnaie des Médailles ${ }^{1}$, qui est née de la scission au milieu du Xvi ${ }^{\mathrm{e}}$ siècle des partisans de la frappe au marteau et des défenseurs de la frappe mécanisée, emménage au rez-de-chaussée de la Grande galerie du palais du Louvre en $1609^{2}$. Du fait de son statut dans l'apanage royal, cette " manufacture " va progressivement développer son savoir-faire pour participer à l'édification de la propagande de Louis XIII. À partir du règne personnel de Louis XIV, l'atelier, qui est constitué d'un directeur ${ }^{3}$ et de quelques ouvriers spécialisés, produit les médailles de son Histoire métallique ${ }^{4}$. À l'époque, l'atelier n'a pas pour mission de garder un exemplaire des médailles et des jetons frappés pour le roi. Ceux-ci sont essentiellement destinés à l'usage privé du souverain qui les offre à ses proches ou les présente dans son Cabinet des médailles personnel à Versailles 5 . En revanche, les poinçons originaux et les matrices sont précieusement conservés depuis l'origine $e^{6}$. Quand Louis XIV nomme Nicolas Delaunay « directeur du balancier des Médailles ", le 24 novembre 1696, son intention est de lui confier la rédaction d'un inventaire descriptif de chaque poinçon et matrice de médaille et jeton. Ce travail de collecte scientifique de données débuta le 9 septembre 1697 et s'acheva le 6 mars $1698^{7}$. Sensible à leur rareté, à la beauté de

1. Jean-Marie Darnis, "Historique du médaillier de la Monnaie de Paris ", Revue belge de numismatique et de sigillographie, t. 133, 1987, p. 179-190 et 192.

2. Pour permettre la construction du Pont Neuf. Cet atelier aussi appelé "Maison des Étuves" était équipé depuis 1552 d'un matériel de fabrication à la pointe de la technologie car entièrement mécanisé.

3. Les directeurs successifs ont été : les membres de la famille Olivier de 1609 à 1629 ; Jean Warin de 1629 à 1636 ; Jean Régnier en 1636 ; Jean Warin de nouveau de 1639 à 1672 ; Isaac Briot en 1643 ; François Warin (le fils de Jean) de 1672 à 1676 ; Claude Ballin de 1676 à 1678 ; l'abbé Bizot de 1678 à 1684 ; Nicolas Petit de 1684 (?) à 1696.

4. Et cela jusqu'à la fin du règne de Louis XVI.

5. Avant que le contenu de ce médaillier ne soit transféré au Cabinet des antiques de la Bibliothèque royale.

6. S'ils n'ont pas été détruits en cours de frappe comme cela se produisait régulièrement. En effet, la frappe au balancier est une technique violente pour les outillages qui reçoivent des chocs de plusieurs tonnes à chaque passe de frappe. Les outillages antérieurs au règne de Louis XIV étaient donc considérés comme des rescapés et, du fait de leur ancienneté, ils possédaient déjà un statut particulier.

7. Jules Guiffrey fut le premier à mentionner la valeur inestimable de cet inventaire dans son étude sur la "Monnaie des Médailles ", Revue numismatique, 1884, p. 484 et années suivantes. À sa suite, 
leur gravure en taille directe (Fig. XVIII, cahier couleur) et la notoriété de leurs créateurs (Fig. XIX, cahier couleur) - tant les dessinateurs que

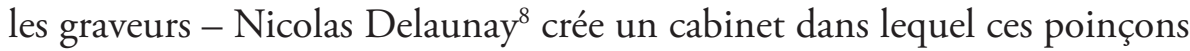
et matrices vont prendre le statut de modèles ; modèles qu'il destine aux artistes et aux visiteurs de marque ${ }^{9}$. Cette initiative intelligente et clairvoyante prouve à quel point les motifs gravés en relief et en creux par les graveurs les plus doués de leur génération ${ }^{10}$ étaient considérés comme de véritables chefs-d'œuvre de la gravure en taille directe. Pour Nicolas Delaunay, il était évident qu'il fallait les préserver et les conserver dans les meilleures conditions ${ }^{11}$. Certes, nous sommes encore loin de la notion de conservation du patrimoine industriel, telle que nous l'entendons du point de vue muséographique, mais il y a là, déjà, une prise de conscience inédite de la part d'un directeur de manufacture royale d'Ancien Régime.

Sous la Révolution française, l'atelier de la Monnaie des Médailles périclite en raison de la cessation des commandes de médailles dites "officielles". Cette mise en sommeil de l'atelier permet opportunément de sauvegarder la collection d'outillages des ambitions de quelques révolutionnaires radicaux qui auraient peut-être bien voulu la détruire, puisqu'elle remémorait l'absolutisme monarchique et véhiculait les emblèmes royaux. Il est vrai que cet ensemble formait une véritable collection " aide-mémoire " dont le caractère archivistique et historique s'était affirmé et amplifié au fil des règnes. Sous le Consulat, la menace étant passée, la volonté de Bonaparte d'utiliser l'art de la médaille comme vecteur de propagande refait surface. La Monnaie des Médailles va définitivement sortir de l'oubli. L'artisan de ce renouveau se nomme Dominique Vivant Denon (1747-1825) ${ }^{12}$. Ayant

Fernand Mazerolle publia l'intégralité de l'« Inventaire des poinçons et des coins de la Monnaie des Médailles (1697-1698) ", Gazette numismatique française, 1903-1907 (tiré à part).

8. Le 3 novembre 1696, Louis XIV crée la charge de conseiller-directeur du «balancier du Louvre». Le premier directeur est l'orfèvre du roi Nicolas Delaunay de 1696 à 1723, puis Jules Robert de Cotte de 1723 à 1737 et Jules François de Cotte de 1767 à 1803.

9. Tel le tsar Pierre Ir qui visita l'atelier en 1717 et se fit remettre une médaille en or, gravée à son effigie par Jean Duvivier.

10. Joseph Roëttiers, Jean Warin, Jean Mauger, Hercule le Breton, Jérôme Roussel, Jean Dollin, Thomas Bernard.

11. A contrario, la destruction des outillages monétaires est une réalité qu'il faut replacer dans un contexte de sauvegarde de souveraineté du monnayage mis en circulation.

12. Denon fut le directeur de la Monnaie des Médailles de 1803 à 1816. Catherine Delmas, "Denon directeur de la Monnaie de Médailles ", Dominique-Vivant Denon l'œeil de Napoléon, Paris, musée du Louvre 20 octobre 1999-17 janvier 2000, RMN, 1999, p. 276-293. Pierre Lelievre, 
la toute confiance du Premier consul, ce dernier le charge de rétablir la Monnaie des Médailles en $1804^{13}$ et d'y conduire la réalisation de son histoire métallique. Comme au temps du "Roi-Soleil ", les médailles sont frappées à la chaîne et les poinçons et les matrices vont très vite se multiplier et s'accumuler sur les étagères de l'atelier ${ }^{14}$. Mais le palais du Louvre, transformé en musée depuis 1793, a besoin d'espace pour y présenter ses chefs-d'œuvre et les nombreuses prises de guerre ramenées par l'empereur à la suite de ses conquêtes. C'est pourquoi en application du décret impérial du 5 mars 1806, la Monnaie des Médailles, devenue trop encombrante avec ses imposants balanciers et ses centaines d'outillages de frappes anciens et récents, est transférée à l'Hôtel des Monnaies, côté rive gauche de la Seine. Là, on lui attribue quelques espaces donnant sur la rue Guénégaud ${ }^{15}$. Pour la seconde fois de son histoire l'atelier déménage et, ironie de l'histoire, le voici proche de son emplacement d'origine, non loin du Pont Neuf! Sous la Restauration, les De Puymaurin père et fils ${ }^{16}$ en développent les activités grâce aux talents des graveurs et des ouvriers, hautement qualifiés. Toutes les forces vives se mobilisent pour répondre aux demandes croissantes des commanditaires publics et privés. En effet, l'art de la médaille se démocratise chaque jour davantage. La renommée de l'atelier dépasse maintenant les frontières de l'hexagone car, grâce à ses productions de qualité, il occupe une place de choix auprès des amateurs étrangers. Mais pour l'instant, le sort du fonds de poinçons et de matrices hérités de l'Ancien Régime n'est pas encore scellé.

\footnotetext{
"Dominique Vivant-Denon égyptologue et directeur de la Monnaie des Médailles ", L'Institut et la Monnaie, deux palais sur un quai, Délégation à l'action artistique de la Ville de Paris, 1990, p. 153-157.

13. Arrêté du 5 germinal an XII, soit le 26 mars 1804.

14. Le graveur Jean-Pierre Droz gérait l'atelier. Il devint aussi le premier conservateur du " Musée des coins".

15. "La Monnaie de Paris ", L'Institut et la Monnaie, deux palais sur un quai, Délégation à l'action artistique de la Ville de Paris, 1990, p. 159-242.

16. Après Denon, les autres directeurs de l'atelier seront : Marcus de Puymaurin (père) de 1816 à 1824 ; Aimé de Puymaurin (fils) de 1824 à 1830 ; A. Clément en 1831. Jean Mazard, "Les De Puymaurin, directeurs de la Monnaie du Roy ", Le Club français de la médaille, bulletin n 15 , 1967, p. 6-8.
} 


\section{L'ordonnance du 24 mars 1832 et son impact sur l'avenir du fonds d'outillages}

En 1827, se dessine l'idée de créer un musée en l'Hôtel des Monnaies. Le 12 septembre, dans une longue lettre, Jean-Baptiste Henry Collin (17761837), comte de Sussy ${ }^{17}$, président de la Commission des Monnaies, expose son dessein au ministre des Finances, Joseph de Villèle (1773-1854). Il y évoque les questions monétaires sans faire mention, toutefois, des outillages de médailles et de jetons. Et non, ces instruments ne sont pas concernés par son projet car l'atelier de la Monnaie des Médailles est indépendant de la Commission des Monnaies qui surveille les ateliers de frappes monétaires pour le compte de l'État. Seuls les instruments de garantie employés à la marque des matières d'or et d'argent le sont. Mais cinq ans plus tard, la situation va changer. Par ordonnance royale du 24 mars $1832^{18}$, la Monnaie des Médailles est réunie à la Commission des Monnaies ${ }^{19}$. La nouvelle entité créée dépend maintenant du ministère des Finances. Cette décision résulte en partie du fait que le roi Louis-Philippe ne voulait plus conserver dans sa liste civile l'atelier en charge de la frappe des médailles de son règne. En procédant ainsi, le roi rompt avec la tradition multiséculaire de ses prédécesseurs qui avaient maintenu l'art de la médaille sous " haute protection » royale. Cet acte démocratique et libéral va engendrer le fait que les poinçons et les matrices hérités de l'ancien atelier de la Monnaie des Médailles vont être intégralement reversés au nouveau Musée monétaire. Ils vont former le premier noyau de collections techniques de la Monnaie de Paris, aux côtés des outillages monétaires et des maquettes de machines. Aménagé dans les salons d'honneur, le Musée monétaire est inauguré le 8 novembre 1833 en présence de la famille royale ${ }^{20}$ (Fig. XX, cahier couleur). Pour l'occasion, un exemplaire des médailles des histoires métalliques

17. L'inspecteur général des Essais, Jean-Pierre Joseph d'Arcet, fut aussi partie prenante dans ce projet concernant les questions monétaires.

18. L'article 6 de la présente ordonnance stipule que le " Musée monétaire » reçut la collection de coins et de matrices ainsi que tous les instruments de fabrication des médailles.

19. En exécution de la loi du 2 mars courant.

20. Le règne de Louis-Philippe fut propice à la création des musées. Grâce aux enseignements fondateurs de Madame de Genlis, le futur roi des Français sut mettre à profit les réflexions de sa préceptrice en matière de pédagogie par l'objet pour promouvoir la création de lieux dédiés à l'histoire, à l'histoire de l'art et des techniques. C'est ainsi que l'idée de créer un musée à l'Hôtel des Monnaies, sous la tutelle de la Commission des Monnaies, a pu se concrétiser. 
des souverains d'Ancien Régime a été spécialement refrappé en cuivre et exposé dans des vitrines plates pour rappeler les grandes dates de l'Histoire de France. Les poinçons et les matrices sont exposés, quant à eux, dans des vitrines murales aménagées dans la bibliothèque ${ }^{21}$. La mission principale de ce musée est de servir de vitrine diplomatique pour promouvoir les savoir-faire artistiques et techniques des ateliers de fabrication. Le musée n'a pas la vocation de promouvoir la recherche sur les objets qui y sont présentés ${ }^{22}$.

Cette collection inestimable, constituée de 3129 poinçons et matrices de médailles datées de 1495 à 1792 a été récolée en $2016^{23}$. La base de données a été enrichie de nombreuses informations techniques (poids, mesures, marques), formant ainsi un outil de travail particulièrement précis et performant. Une campagne photographique intégrale a été menée en même temps, consistant en une prise de vue de chaque face d'un même outillage pour en relever les numéros d'inventaires anciens qui y ont été gravés au fil des siècles. Enfin, lors de la préparation des salles du nouveau musée du 11 Conti, il a été décidé d'offrir une place de choix à cette collection unique en exposant un échantillon de soixante-dix outillages représentatifs en termes artistiques (qualité de la gravure, signatures célèbres, iconographies) et techniques (alliages, évolution de la forme des poinçons et matrices). Cette présentation didactique est aujourd'hui une des attractions les plus appréciées du public qui peut bénéficier, chaque mercredi, d'une démonstration de gravure ${ }^{24}$ pour lui donner à comprendre les gestes de cet art majeur pratiqué depuis des siècles à la Monnaie de Paris.

21. Ils y sont restés jusqu’au milieu des années 1970.

22. Il n'était accessible que sur autorisation exceptionnelle.

23. La collection de poinçons et de matrices de jetons est actuellement en cours d'étude.

24. La gravure en taille directe est toujours pratiquée à la Monnaie de Paris. 